\title{
On a class of metric spaces admitting simply transitive groups of motions.
}

\author{
By JACK Levine (to Raleigh, N. C. - (U. S. A.))
}

1. Introduction. - It has been shown (1) that if a metric space, $V_{n}$, admits a simply transitive group of motions, then it also admits an orthogonal ennuple whose Rroor coefficients of rotation ( $\left.{ }^{*}\right), \gamma_{i, j}$, are constants, and conversely. We investigate here the metric spaces $V_{n}$ admitting an $n$-tuply orthogonal system of hypersurfaces such that the $\gamma_{i j k}$ associated with the normal congruences $\left({ }^{3}\right)$ are constant. Such $V_{n}$, by the above theorem, will then admit simply transitive groups of motions.

2. The General Case. - If in the coordinate system in which the congruences of the normal ennuple are taken as parametric we put

then we have $\left({ }^{4}\right)$

$$
g_{i, j}=e_{i} \delta_{j}^{i} H_{i}^{2}, \quad\left(e_{i}= \pm 1\right),
$$

$$
e_{i} \gamma_{h i i}=\frac{1}{H_{h} \bar{H}_{i}} \frac{\partial H_{i}}{\partial x^{h}}, \quad(h \neq i)
$$

the remaining $\gamma$ 's being zero. Geometrically, this means that the radii of principal normal curvature of the orthogonal hypersurfaces are constant. If $r_{i j}$ is the radius of principal normal curvature of $x^{i}=$ const. for the curve of parameter $x^{j}$, then ( $\left.{ }^{5}\right)$

$$
\frac{1}{r_{i j}}=-e_{j} \gamma_{i, j j}
$$

We wish to solve (2.2) for the $H_{i}$ assuming the $\gamma$ 's are constants. We

() G. D. Matroul, «Atti del R. Istituto Veneto", 89, pp. 97-105 (1929). G. VRaNCEANU, «Comptes Rendus", 189, pp. 386-389 (1929).

(2) L. P. Ersenhart, Riemannian Geometry, p. 97. We refer to this book as RG.

(3) RG, p. 114 .

(4) $\mathrm{BG}, \mathrm{p}, 119$.

(5) L. Branchi, Lezioni di Geometria differenziale, vol. 2, p. 472 (1923). 
put $a_{i n}=c_{i} \gamma_{h i i}$, and (2.2) can then be written

$$
\frac{\partial H_{i}}{\partial x^{h}}=a_{i n} H_{h} H_{i}, \quad(h \neq i) .
$$

The integrability conditions of (2.3) are

$$
a_{i h} a_{h, j}-a_{i j} a_{j h}=0, \quad(h, i, j \neq) .
$$

(We assume $n>2$, the case $n=2$ being treated later). We shall consider several cases depending on the number of $a_{i j}$ which are zero.

CASE I. - All $a_{i j} \neq 0$.

From (2.4) we then have

$$
\frac{a_{i h}}{a_{j h}}=\frac{a_{i, j}}{a_{h j}}, \quad \frac{a_{h i}}{a_{j i}}=\frac{a_{h, j}}{a_{i, j}}
$$

So

$$
\frac{a_{j h}}{a_{i n}}=\frac{a_{j i}}{a_{h i}}=\frac{a_{i j}}{a_{h j}}=\frac{a_{i h}}{a_{j h}}
$$

which gives $a_{i n}^{2}=a_{j h}^{2}$, or

$$
a_{i h}= \pm a_{j n}, \quad(h, i, j \neq) .
$$

We can therefore put $a_{i, j}= \pm a$, where $a_{j}$ are $n$ non-zero constants. We shall now show that for $n>3$, we must ase the plus sign only, i. e., $a_{i j}=+a_{j}$. Consider then any three of the $a_{i n}$ for $h$ fixed, say, $a_{i n}, a_{j h}, a_{k h},(h, i, j, k \neq)$, and suppose $a_{l h}=a_{j h}=-a_{k h}$, (two of the $a^{\prime} s$ must have the same sign). Then

Hence

$$
\begin{gathered}
\frac{a_{i h}}{a_{j h}}=\frac{a_{j h}}{a_{i h}}=\frac{a_{i j}}{a_{h i}}=1, \\
\frac{a_{i h}}{a_{k h}}=\frac{a_{k h}}{a_{i h}}=\frac{a_{k i}}{a_{h i}}=\frac{a_{i k}}{a_{h k}}=\frac{a_{j h}}{a_{k h}}=\frac{a_{j k}}{a_{h k}}=-1 .
\end{gathered}
$$

$$
\frac{a_{j k}}{a_{k}}=\frac{a_{j i}}{a_{k i}}=1
$$

or $a_{j i}=a_{h i}=a_{k i}$ from the first and third sets of equations and $a_{k i}=-a_{h i}$ from the second. This gives a contradiction to $\alpha_{i j} \neq 0$, and so $\alpha_{i j}=a_{j}$. The system (2.3) thus becomes

$$
\frac{\partial H_{i}}{\partial x^{h}}=a_{h} H_{h} H_{i}, \quad(h \neq i)
$$

By expressing the components $R_{h i j k}$ of the curvature tensor in terms of the $H_{i}$ and their derivatives and then substitating for these derivatives by 
means of (2.5) it is found that the $V_{n}$ determined by the $H_{i}$ is of constant curvature $\left({ }^{6}\right)$. We state this result in the following form.

If a $\mathrm{V}_{\mathrm{n}},(\mathrm{n}>3)$, admits an $\mathrm{n}$-tuply orthogonal system of hypersurfaces whose radii of principal normal curvature are non-zero constants, the $\nabla_{\mathrm{n}}$ is of constant curvature.

It is consequently unnecessary to solve (2.5), but as similar equations are encountered in later cases we give here briefly the solution of (2.5) for

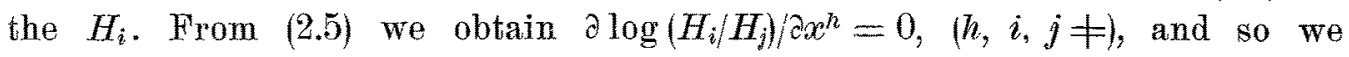
can write $(?)$

$$
\begin{aligned}
\log \frac{H_{i}}{H_{j}}=\theta_{i j}\left(x^{i}, x^{j}\right) & =-\theta_{i j}, & (i \neq j), \\
\theta_{i j}+\theta_{j k}+\theta_{k i} & =0, & (i, j, k \neq) .
\end{aligned}
$$

On differentiating this last equation with respect to $x^{i}$ and $x^{j}$ we obtain $\partial^{i} \theta_{i j} / \partial x^{i} \partial x^{j}=0$ the solutions of which can be written $\theta_{i j}=\theta_{i}\left(x^{i}\right)-\theta_{j}\left(x^{j}\right)$. We then obtain $H_{i}{ }^{\prime} \eta_{i}\left(x^{i}\right)=H_{j} / \eta_{j}\left(x^{j}\right)=\tau$, say, or $H_{i}=\tau \eta_{i}$. Substituting these values for $H_{i}$ in $(2.5)$ gives $\tau^{-1}=-\sum_{i} a_{i} \int \eta_{i} d x^{i}$, and

$$
H_{i}=-\frac{1}{a_{i}} \frac{\Phi_{i}^{\prime}}{\Sigma \Phi_{j}\left(x^{j}\right)}
$$

where

$$
\Phi_{i}\left(x^{i}\right)=a_{i} \int \eta_{i} d x^{i}
$$

Under the coordinate transformation $\bar{x}^{i}=\Phi_{i} / a_{i}$, the $\bar{g}_{i j}$ have the form

$$
\vec{g}_{i j}=\frac{e_{i} \delta_{j}^{i}}{\left(\sum_{h} a_{f_{t}} \overline{\left.x^{n}\right)^{2}}\right.},
$$

this being one of the forms for the metric of a $V_{n}$ of constant curvature $K$ which in this case is $\left({ }^{8}\right) K=-e_{i} a_{i}{ }^{2}$.

3. Special Cases. - In the following cases to be considered we assume some of the $\alpha_{i j}=0$, and that at least two of the $H$ 's are equal. Without this last assumption there appears to be no systematic method for imposing

(6) RG, pp. 84, 119.

(7) Tronghout this paper all functions are to be analytic in their arguments, and an expression like $\Phi_{i}\left(x^{i}\right)$ indicates $\Phi_{i}$ is a function of the variable $x^{i}$ only.

(8) RG, p. 85 . 
restrictions on the $a_{i j}$ other than those of case I. We thus put

$$
H_{1}=H_{2}=\ldots=H_{r}=H, \quad(2 \leqq r \leqq n-2),
$$

(the cases $r=n \cdots 1$ and $r=n$ are treated later). We let small Greek letters have the range $1, \ldots, r$. If in $(2.3)$ we take $i=\alpha$, we get

$$
\frac{1}{H H_{h}} \frac{\partial H}{\partial x^{h}}=a_{x h} \equiv a_{h}
$$

since the first member of the above equation is independent of $\alpha$. We now let large Latin letters have the range of all indices for which $a_{n} \neq 0$, and small Latin letters from a through $g$ the range for which $a_{n}=0$, (in both cases the $a_{\alpha}$ are not to be included), the indices from $h$ through $z$ are to have the range $1, \ldots, n$. We thus divide the $a_{n}$ in three groups, $a_{\alpha}, a_{I}, a_{b}=0$. If there are $t$ indices in the range for $\alpha_{I}$, and $s$ in the range for $a_{b}$, then $r+s+t=n$.

We now divide the system (2.3) into nine sets
(a) $\frac{1}{H^{2}} \frac{\partial H}{\partial x^{\alpha}}=a_{x}$,
(d) $\frac{1}{H H_{I}} \frac{\partial H_{I}}{\partial x^{x}}=a_{I x}$,
(g) $\frac{1}{H H_{b}} \frac{\partial H_{b}}{\partial x^{2}}=a_{b \alpha}$,
(b) $\frac{1}{H H_{I}} \frac{\partial H}{\partial x^{I}}=a_{I}$,
(e) $\frac{1}{H_{I} H_{J}} \frac{\partial H_{J}}{\partial x^{I}}=a_{J I},(I \neq J)$,
(h) $\bar{H}_{b} \frac{1}{H_{\mathrm{c}}} \frac{\partial H_{b}}{\partial x^{c}}=a_{b e},(b \neq c)$,
(c) $\frac{1}{H H_{a}} \frac{\partial H}{\partial x^{\mu}}=0$,
(f) $\frac{1}{H_{b} H_{J}} \frac{\partial H_{J}}{\partial x^{b}}=a_{J b}$,
(i) $\frac{1}{H_{I} H_{b}} \frac{\partial H_{b}}{\partial x^{I}}=a_{b I}$.

If $s$ or $t$ is 0 or 1 certain of the above equations will not be present.

CASE IIa. - At least one $a_{\alpha} \neq 0, t>1, s>1, a_{b c} \neq 0$.

By a proper choice of the indices in (2.4) we find

$$
a_{I \alpha}=a_{\alpha}, \quad a_{I J}=a_{J}, \quad a_{A b}=a_{b \alpha}=a_{b I}=0 .
$$

These values are to be substituted in the above sets. From (a) and (c) we get $H=1 /\left(f\left(x^{I}\right)-a_{x} x^{x}\right)$, where $f$ is to be determined. Then from (b) we have

$$
H_{I}=-\frac{1}{a_{I}} \frac{1}{f-a_{x} x^{\alpha}} \frac{\partial f}{\partial x^{I}},
$$

which satisfies (d) identically. Substituting for $H_{I}$ in (e) gives $\partial^{2} f / \partial x^{I} \partial x^{J}=0$, or $f=\underset{I}{\Sigma} \Phi_{I}\left(x^{I}\right)$. Hence

$$
H=\frac{1}{\Sigma \Phi_{I}\left(x^{I}\right)-a_{x} x^{x}}, \quad H_{I}=-\frac{1}{a_{I}} \frac{\Phi_{I}^{\prime}}{\Sigma \Phi_{J}-a_{x} x^{\nu}} .
$$

From (g) and (i) we see $\Pi_{a}=H_{a}\left(x^{\prime}\right)$, and (h) are exactly the same form 
as (2.3). Hence if $s>3$ we may put $a_{b c}=\alpha_{c}$, and then

$$
H_{a}=-\frac{1}{\alpha_{a}} \frac{\Phi_{a}^{\prime}}{\Sigma \Phi_{b}\left(x^{b}\right)}
$$

If $s=2$ or $s=3$ the values of the $H_{a}$ will be the same as for the $H_{i}$ for $n=2$ and $n=3$, these cases are treated later.

CASE IIb. - At least one $a_{b} \neq 0, t>1, a_{b c}=0$.

From (2.4) we obtain

$$
a_{I \alpha}=a_{\alpha}, \quad a_{I J}=a_{J}, \quad a_{A b}=0, \quad \frac{a_{b J}}{a_{J}}=\frac{a_{b I}}{a_{I}} \equiv-\rho_{b}=\frac{a_{b_{\alpha}}}{a_{\alpha}}, \quad\left(\text { if } \quad a_{\alpha} \neq 0\right) .
$$

Using these values in (a)-(i) we find $H$ and $H_{I}$ have the same values as for case IIa. From (g) and (i) we then obtain

$$
\left.H_{a}=\left[\underset{I}{\Sigma} \Phi_{T}\left(x^{I}\right)-a_{a} x^{\alpha}\right]\right]_{\alpha} \Phi_{a}\left(x^{a}\right) .
$$

In this case, the hypersurfaces $x^{a}=$ const. are totally geodesic $\left({ }^{9}\right)$.

CASE IIIa. - All $a_{\alpha}=0, t>1, s>1, a_{b c} \neq 0, a_{I J} \neq 0$.

From (2.4) we obtain

$$
a_{I \alpha}=a_{I a}=a_{b x}=a_{b A}=0 .
$$

From (a) and (c) we then get $H=1 / f\left(x^{A}\right)$, which when substituted in (b) gives

$$
H_{A}=-\frac{1}{a_{A}} \frac{\partial \sigma}{\partial x^{A}}, \quad(\sigma=\log f) .
$$

And this value used in (e) gives

$$
\frac{\partial^{2} \sigma}{\partial x^{A} \partial x^{\bar{B}}}=P_{A B} \frac{\partial \sigma}{\partial x^{A}} \frac{\partial \sigma}{\partial x^{B}}, \quad(A+B),
$$

where

$$
\rho_{A B}=\rho_{B A}=-\frac{a_{B A}}{a_{A}}=-\frac{a_{A B}}{a_{B}} .
$$

The integrability conditions of these equations in $\sigma$ are

i. e.,

$$
P_{B C}\left(P_{A B}-P_{A C}\right)=0, \quad(A, B, C \neq),
$$

and $\rho \neq 0$ since $a_{I J} \neq 0$.

$$
P_{A B}=P_{A C}=\rho, \text { say, }
$$

If we place $\sigma=\frac{1}{\rho} \log \frac{c}{\tau}$, where $c$ is a constant, (3.1) reduces to $\partial^{2} \tau ; \partial x^{A} \partial x^{B}=0$.

(9) RG, p. 183. 
Hence

which gives

$$
\sigma=\frac{1}{\rho} \log \frac{c}{\Sigma \Phi_{A}\left(x^{A}\right)}
$$

$$
H=\frac{1}{k_{k}}\left[\Sigma \Phi_{J}\left(x^{I}\right)\right]^{1}, \quad H_{I}=\frac{1}{\rho a_{I}} \frac{\Phi_{I}^{\prime}}{\Sigma \Phi_{J}}, \quad(k=\text { const. })
$$

If $t=2$ we ean put $\rho_{A B}=\rho$ in (3.1) and proceed as above. If $s>3$ we may put $a_{b c}=a_{c}$; and obtain

$$
H_{a}=-\frac{1}{\alpha_{a}} \frac{\Phi_{a}^{\prime}}{\Sigma \Phi_{b}\left(x^{b}\right)},
$$

$x^{x}=$ const. are totally geodesic.

CASE IIIb. - All $a_{\alpha}=0, t>1, a_{b c}=0, a_{I J} \neq 0$.

From (2.4) we have $a_{1 \alpha}=a_{1 b}=a_{b \alpha}=0$.

It is seen that $H$ and $H_{I}$ have the same form as in IIIa. From (i) we then get

$$
H_{a}=\left(\Sigma \Phi_{I}\right)^{-\frac{\rho_{a}}{\rho}} \Phi_{a}\left(x^{a}\right)
$$

where $\rho_{a}$ and $\rho$ are defined as in $11 b$ and IIIa.

CASE IIIC. - All $a_{x}=0, t>1, s>1, \alpha_{b c} \neq 0, a_{I J}=0$.

We proceed as in IIIa. Now $\rho=0$ and (3.1) gives $\sigma=\Sigma \omega_{X}$, so

$$
H=e^{-\Sigma \Phi_{I}(x I)}, \quad H_{I}=-\frac{\Phi_{I}^{\prime}}{a_{I}} .
$$

If $s>3$, we have the same solutions for $H_{a}$ as in IIIa. For $s=2$ or $s=3$ we use the forms as given in the cases $n=2$ and $n=3$.

CASE IIId. - All $a_{\alpha}=0, t>1, a_{b c}=a_{I J}=0$.

We find $H$ and $H_{Y}$ have the same form as for IIIc. From (1) we then get

$$
H_{a}=e^{\rho_{a}} \Sigma \Phi_{I(x)} \Phi_{a}\left(x^{a}\right) \text {. }
$$

If in IIa, IIb, IIIc, and IIId we replace the condition $t>1$ by $t=0$ or $t=1$, it is found that the $H^{\prime}$ 's have the same form as for $t>1$ (with one exception). For $t=0$ we replace $\Sigma \Phi_{I}$ by a constant. The exception occurs in IIId when we use $t=0$. The value of $H_{a}$ is given by

$$
H_{a}=e^{\frac{1}{\bar{k}_{\alpha}} \Sigma a_{a \alpha x \alpha}} \Phi_{a}\left(x^{a}\right) .
$$

If $r=n-1$, we have the equations

$$
\frac{1}{H^{2}} \frac{\partial H}{\partial x^{\alpha}}=a_{\alpha}, \quad \frac{1}{H H_{n}} \frac{\partial H_{n}}{\partial x^{\alpha}}=a_{n \alpha}, \quad \frac{1}{H H_{.,}} \frac{\partial H}{\partial x^{n}}=a_{n} .
$$


If $a_{n} \neq 0$, we obtain

$$
H=\frac{1}{\Phi\left(x^{n}\right)-a_{\alpha} x^{\alpha}}, \quad H_{n}=\frac{-\Phi^{\prime}}{a_{n}\left(\Phi-a_{\alpha} x^{\alpha}\right)} .
$$

If $a_{n}=0$, and at least one $a_{\alpha} \neq 0$,

$$
H=\frac{1}{k-a_{\alpha} x^{x}}, \quad H_{n}=\left(k-a_{\alpha} x^{\alpha}\right)^{\rho_{n}} \Phi\left(x^{n}\right), \quad \rho_{n}=-\frac{a_{n \alpha}}{a_{\alpha}}, \quad\left(\text { for } a_{\alpha} \neq 0\right) .
$$

If $a_{n}=\alpha_{\alpha}=0$,

$$
H=\frac{1}{k}, \quad H_{n}=e^{\frac{1}{k} \Sigma a_{\alpha} x_{\alpha} x^{\alpha}} \Phi\left(x^{n}\right)
$$

If $r=n$, we have one equation

$$
\frac{1}{H^{2}} \frac{\partial H}{\partial x^{i}}=a_{i}
$$

or

$$
H=\frac{1}{k-a_{i} c^{i}}
$$

The space is hence of constant curvature, which proves the theorem.

If a conformally fat space admits an ennuple of normal congruences with constant Ricci coefficients, the space is of constant curvature.

4. The case $\|=2$. - For this case $(2.3)$ reduce to

$$
\frac{\partial H_{1}}{\partial y}=a_{12} H_{1} H_{2}, \quad \frac{\partial H_{2}}{\partial x}=\alpha_{21} H_{1} H_{2}, \quad\left(x^{1}=x, x^{2}=y\right) .
$$

The resulting space is always of constant curvature. If $a_{12} \neq 0, a_{31} \neq 0$, we obtain

If $a_{12}=0, a_{21} \neq 0$,

$$
H_{1}=-\frac{1}{a_{21}} \frac{\Phi_{1}{ }^{\prime}(x)+\Phi_{2}(y)}{\Phi_{1}}, \quad H_{2}=-\frac{1}{a_{12}} \frac{\Phi_{2}{ }^{\prime}}{\Phi_{1}+\Phi_{2}} .
$$

If $a_{12}=a_{21}=0$,

$$
H_{1}=\Phi_{1}^{\prime}(x), \quad H_{2}=e^{a_{21} \Phi_{1}(x)} \Phi_{2}(y) .
$$

$$
H_{1}=\Phi_{1}(x), \quad H_{2}=\Phi_{2}(y)
$$

5. The case $n=3$. - If all $a_{i j} \neq 0$, we must have, as has been shown, $a_{i j}= \pm a_{j}$. If $a_{i j}=+a_{j}$, the space is of constant curvature and the $H$ 's have the same form as for case $\mathrm{I}$. We consider the case where some $a_{i j}=+a_{j}$, and the remaining $a_{i j}=-a_{j}$, later. There are three distinct cases if some 
$a_{i j}=0$. Let $h, i$, and $j$ have the values $1,2,3$ in some order. Then the three cases are given by

$$
\begin{aligned}
& a_{i h}=a_{j h}=0, \quad a_{i j} \neq 0, \quad a_{j i} \neq 0, \\
& a_{i h}=a_{j h}=a_{i j}=a_{h j}=0, \quad a_{j i} \neq 0, \\
& a_{i h}=a_{j h}=a_{i j}=a_{j i}=0 .
\end{aligned}
$$

We find for $(\alpha)$

$$
H_{i}=-\frac{\Phi_{i}^{\prime}}{a_{j i}\left[\Phi_{i}\left(x^{i}\right)+\Phi_{j}\left(x^{j}\right)\right]}, \quad H_{j}=-\frac{\Phi_{j}^{\prime}}{a_{i j}\left(\Phi_{i}+\Phi_{j}\right)}, \quad H_{h}=e^{o_{k}}\left(\Phi_{i}+\Phi_{j}\right) \Phi_{h}\left(x^{h}\right),
$$

where

For $(\beta)$,

$$
\rho_{h}=-\frac{a_{h i}}{a_{j i}}=-\frac{a_{h j}}{a_{i j}}
$$

$$
H_{i}=\Phi_{i}^{\prime}, \quad H_{j}=e^{a_{j i} \Phi_{i}\left(x^{i}\right)} \Phi_{j}\left(x^{j}\right), \quad H_{h}=e^{a_{k i} \Phi_{i} \Phi_{h}\left(x^{h}\right)}
$$

For $(\gamma)$,

$$
H_{i}=\Phi_{i}\left(x^{i}\right), \quad H_{j}=\Phi_{j}\left(x^{j}\right), \quad H_{h}=e^{a_{k i} \Phi_{i}+a_{k j} \Phi_{i}} j \Phi_{h}\left(x^{h}\right) .
$$

For the case $a_{i j} \neq 0$, and $a_{i j} \neq+a_{j}$, we have only one other possibility

We put

$$
a_{12}=-a_{32} \equiv a, \quad a_{13}=-a_{23} \equiv b, \quad a_{21}=-a_{31} \equiv c .
$$

$$
H_{1}=u, \quad H_{2}=v, \quad H_{3}=w, \quad x^{1}=x, \quad x^{2}=y, \quad x^{3}=z
$$

Then our equations (2.3) become

$$
\begin{array}{ll}
\frac{\partial u}{\partial y}=a u v, & \frac{\partial v}{\partial x}=c u v \\
\frac{\partial u}{\partial z}=b u w, & \frac{\partial w}{\partial x}=-c u w \\
\frac{\partial v}{\partial z}=-b v w, & \frac{\partial w}{\partial y}=-a v w .
\end{array}
$$

We need only consider (5.1) and (5.2) since (5.3) follow from them as is easily shown. If we solve the first equations in (5.1) and (5.2) for $v$ and $w$ in terms of $u$, substitute in the second and put $u=e^{-h}$ in the results we obtain

$$
\frac{\partial^{2} h}{\partial x \partial y}+c \frac{\partial e^{-h}}{\partial y}=0, \quad \frac{\partial^{2} h}{\partial x \partial z}-c \frac{\partial e^{-h}}{\partial z}=0
$$

Hence we may write

$$
\frac{\partial h}{\partial x}+c e^{-h}=\Phi_{1}(x, z), \quad \frac{\partial h}{\partial z}-c e^{-h}=\Phi_{2}(x, y) .
$$


From these equations we obtain

$$
e^{-h}=u=\frac{1}{2 c}\left(\Phi_{1}-\Phi_{2}\right), \quad \frac{\partial h}{\partial x}=\frac{1}{2}\left(\Phi_{1}+\Phi_{2}\right)
$$

so that

$$
\frac{\partial \Phi_{1}}{\partial x}-\frac{\partial \Phi_{2}}{\partial x}=\frac{1}{2}\left(\Phi_{1}^{2}-\Phi_{z}^{2}\right)
$$

Differentiating this last equation with respect to $y$ and $z$ gives

$$
\frac{\partial^{2} \Phi_{2}}{\partial x \partial y}+\Phi_{2} \frac{\partial \Phi_{2}}{\partial y}=0, \frac{\partial^{2} \Phi_{1}}{\partial x \partial z}+\Phi_{1} \frac{\partial \Phi_{1}}{\partial z}=0
$$

Hence $\Phi_{1}$ and $\Phi_{2}$ satisfy an equation of the form

$$
\frac{\partial^{2} \Phi}{\partial \alpha \partial \beta}+\Phi \frac{\partial \Phi}{\partial \beta}=0
$$

This equation has for its general solution

$$
\Phi=-\frac{\lambda^{\prime \prime}(\alpha)}{\lambda^{\prime}(\alpha)}+\frac{\lambda^{\prime}}{\frac{1}{2} \lambda(\alpha)+\mu(\beta)},
$$

where $\lambda(\alpha)$ and $\mu(\beta)$ are arbitrary. We thus obtain

$$
u=\frac{1}{2 e}\left[-\frac{\gamma^{\prime \prime}}{\gamma^{\prime}}+\frac{\gamma^{\prime}}{\frac{\gamma(x)}{2}+\mu(z)}+\frac{\delta^{\prime \prime}}{\delta^{\prime}}-\frac{\delta^{\prime}}{\frac{\delta(x)}{2}+\lambda(y)}\right],
$$

and $\gamma(x)$ and $\delta(x)$ must satisfy the relation

$$
\frac{\left(\gamma^{\prime \prime}\right)^{2}+2 \gamma^{\prime} \gamma^{\prime \prime \prime}}{\left(\gamma^{\prime}\right)^{2}}=\frac{\left(\delta^{\prime \prime}\right)^{2}+2 \delta^{\prime} \delta^{\prime \prime \prime}}{\left(\delta^{\prime}\right)^{2}}
$$

This is obtained from the condition (5.4). It is possible to solve the above relation for $\delta$ in terms of $\gamma$ and its derivatives. From (5.1) and (5.2) we then get $v$ and $w$.

If we take $\gamma=\delta$, and make the change of coordinates $x^{\prime}=\gamma(x), y^{\prime}=\lambda(y)$, $z^{\prime}=\mu(z)$, the new $u, v$, and $w$ have the form (dropping primes)

$$
u=\frac{1}{c} \frac{y-z}{(x+y)(x+z)}, \quad v=\frac{1}{a} \frac{x+z}{(y-z)(x+y)}, \quad w=-\frac{1}{b} \frac{x+y}{(y-z)(x+z)} .
$$

6. The Groups. - We shall now obtain the simply transitive groups of motions, $G_{n}$, admitted by the spaces under consideration. Let $\xi_{k \mid}^{i}$ denote the $n$ 
vectors of the $G$ and $\lambda_{h \mid}^{i}$ the components of the normal congruences with the constant $\gamma_{h i j}$. The $\lambda_{h !}^{i}$ are given by $\left({ }^{4}\right) \lambda_{k i}^{i}=\delta_{h \mid}^{i} / H_{i}$. The $\lambda_{h \mid}^{i}$ define the reciprocal group of the $G_{n}^{i}$, and so $\left({ }^{10}\right)$

$$
\xi_{h \mid}^{i} \frac{\partial \lambda_{k \mid}^{j}}{\partial x^{i}}-\lambda_{k p \mid}^{i} \frac{\partial \xi_{h \mid}^{j}}{\partial x^{i}}=0
$$

which because of the form of $\lambda_{h \text { i }}^{i}$ reduce to

$$
\xi_{h \mid}^{i} \frac{\partial \lambda_{h \mid}^{j}}{\partial x^{i}}-\lambda_{k i}^{k} \frac{\partial \xi_{h \mid}^{j}}{\partial x^{k}}=0
$$

If $j \neq k$, it follows that $\partial \xi_{h l}^{j} / \partial x^{k}=0,(j \neq k)$, so $\xi_{h \mid}^{i}=\xi_{h(l)}^{i}\left(x^{i}\right)$. If $j=k$, we obtain

$$
\frac{\partial \xi^{j}}{\partial x^{j}}=-\frac{\partial \log H_{j}}{\partial x^{i}} \xi^{i}
$$

where we have dropped the subscript index on the $\xi$ since these equations are the same for all the $n$ vectors. The equations (6.1) are easy to solve for the $\xi^{i}$. We shall illustrate the method, using case IIb. In (6.1) taking $j=\alpha$ we get

$$
-\frac{d \xi^{\alpha}}{d x^{\alpha}}=\frac{\partial \log H}{\partial x^{\beta}} \xi^{\beta}+\frac{\partial \log H}{\partial x^{I}} \xi^{I}
$$

Since the right member of (6.2) is independent of $\alpha$ we may put $-d \xi^{\alpha} / d x^{\alpha}=k$, where $k$ is a constant, and thus obtain

$$
\xi^{\alpha}=-k c^{\alpha}+k^{\alpha} \text {. }
$$

The $k^{x}$ are also constant. On substituting these values for $\xi^{x}$ back in (6.2) we get

and hence we may set

$$
k \underset{I}{\Sigma} \Phi_{I}+\Phi_{J}^{\prime} \xi^{J}=\alpha_{\beta} c^{\beta}
$$

$$
k \Phi_{I}+\Phi_{I}^{\prime} \xi^{I}=k^{I}(=\text { const. }), \quad \Sigma k^{I}=a_{\beta} c^{3},
$$

since $\Phi_{I}$ and $\xi^{I}$ are functions of $x^{I}$ only. From this last equation, we have

$$
\xi^{I}=\frac{k^{I}-k \Phi_{I}}{\Phi_{I}^{\prime}}
$$

Using the values just found for $\xi^{\alpha}$ and $\xi^{I}$ in (6.1) for $j=J$, we find these equations are identically satisfied. Now in $(6.1)$ put $j=a$, and replace

(10) L. P. Eisenhart, Continuous Groups of Transformations, Princeton University Press, (1933), p. 113. 
$\xi^{\alpha}, \xi^{I}$ by their values. We obtain

$$
\frac{d \xi^{a}}{d x^{a}}=-\frac{a \log \Phi_{a}}{d x^{a}} \xi^{a}+k \rho^{a}
$$

which gives

$$
\xi^{a}=\frac{k^{a}+\rho_{a} k \theta_{a}}{\theta_{a}^{\prime}}, \quad\left(\theta_{a}^{\prime}=\Phi_{a}, k^{a}=\text { const. }\right) .
$$

The form of the $\xi$ 's for the other eases are given as follows.

CASE IIa. - $(s>3)$,

$$
\begin{gathered}
\xi^{\alpha}=-k x^{\alpha}+k^{\alpha}, \quad \xi^{I}=\frac{k^{I}-k \Phi_{I}}{\Phi_{I}^{\prime}}, \quad \xi^{a}=\frac{l \Phi_{a}-k^{a}}{\Phi_{a}^{\prime}}, \\
\Sigma k^{a}=0, \quad \Sigma k^{I}=a_{\alpha} c^{\alpha}, \quad(k, l=\text { const. }) .
\end{gathered}
$$

CAsE IIIa. - Same as for IIa.

CASE IIIb. - Same as for IIb.

CASE IIIc. - $(s>3)$,

$$
\begin{gathered}
\xi^{a}=-k x^{\alpha}+k^{\alpha}, \quad \xi^{I}=-\frac{k^{I}}{\Phi_{I}^{\prime}}, \quad \xi^{a}=\frac{l \Phi_{a}-k^{a}}{\Phi_{a}^{\prime}}, \\
\Sigma k^{a}=0, \quad \Sigma k^{I}=-k .
\end{gathered}
$$

CASE IIId.

$$
\begin{gathered}
\xi^{\alpha}=-k x^{\alpha}+k^{\alpha}, \quad \xi^{I}=-\frac{k^{I}}{\Phi_{I}^{\prime}}, \quad \xi^{a}=\frac{k^{a}-k \rho_{a} \theta_{a}}{\theta_{a}^{\prime}}, \\
\Sigma k^{I}=-k, \quad \Phi_{a}=\theta_{a}^{\prime} .
\end{gathered}
$$

In those cases for which $H=$ const., $k=0$. In IIId for $t=0$ we get

$$
\xi^{\alpha}=k^{\alpha}, \quad \xi^{b}=\frac{k^{b}-\frac{1}{l} k^{\alpha} a_{b \alpha} \theta_{b}}{\theta_{b}^{\prime}}, \quad\left(H=\frac{1}{l}, \Phi_{b}=\theta^{\prime}{ }_{b}\right) .
$$

The forms of the $\xi$ 's for the remaining cases are easily obtained. They are essentially the same as those given.

Note added in proof: While this paper was in the hands of the editors, a paper by L. P. Eisenhart appeared on the same problem ("Monatsheften für Math. und Physik", vol. 43). From his results, it is seen that $\gamma_{h i, j}=$ const. is the general condition for the spaces under consideration. Also the condition that at least two $H$ 's be equal constitutes no real restriction. 